\title{
PI3K/Akt and Stat3 signaling regulated by PTEN control of the cancer stem cell population, proliferation and senescence in a glioblastoma cell line
}

\author{
SEOK-HO MOON, DAE-KWAN KIM, YOUNG CHA, IKSOO JEON, JIHWAN SONG* and KYUNG-SOON PARK* \\ Department of Biomedical Science, College of Life Science, CHA University, Seoul, Republic of Korea
}

Received May 15, 2012; Accepted July 4, 2012

DOI: $10.3892 /$ ijo.2013.1765

\begin{abstract}
Malignant gliomas are the most common primary brain tumor in adults. A number of genes have been implicated in glioblastoma including mutation and deletion of PTEN. PTEN is a regulator of PI3K-mediated Akt signaling pathways and has been recognized as a therapeutic target in glioblastoma. To achieve potent therapeutic inhibition of the PI3K-Akt pathway in glioblastoma, it is essential to understand the interplay between the regulators of its activation. Here, ectopic expression of PTEN in the U-87MG human glioblastoma-astrocytoma cell line is shown to result in the depletion of glioblastoma stem cells (GSCs) and to cause growth retardation and senescence. These effects are likely to be associated with PTEN-mediated cooperative perturbation of Akt and Stat 3 signals. Using an in vivo rat model of glioblastoma, we showed that PTEN-overexpressing U-87MG cells failed to induce tumor formation, while untreated U-87MG cells did so. Furthermore, cells expressing the phosphorylated form of Stat3 were completely absent from the brain of rats implanted with PTEN-overexpressing U-87MG cells. Based on these results, PTEN appears to function as a crucial inhibitor of GSCs and as an inducer of senescence, suggesting that functional enhancement of the PTEN pathway will be useful to provide a therapeutic strategy for targeting glioblastoma.
\end{abstract}

Correspondence to: Professor Kyung-Soon Park, Department of Biomedical Science, College of Life Science, CHA University, 222 Yatap-dong, Bundang-gu, Seongnam-si, Gyeonggi-do 463-836, Republic of Korea

E-mail:kspark@cha.ac.kr

Dr Jihwan Song, Department of Biomedical Science, College of Life Science, CHA University, 606-16 Yeoksam 1-dong, Kangnam-gu, Seoul 135-081, Republic of Korea

E-mail: jsong@cha.ac.kr

${ }^{*}$ Contributed equally

Key words: PTEN, cancer stem cell, Stat3, Akt, senescence, proliferation

\section{Introduction}

Phosphatase and tensin homolog $(P T E N)$ is one of the most frequently lost or mutated of the tumor suppressor genes (1-5). It has a structural motif for a dual specificity protein phosphatase, suggesting that $P T E N$ functions to negatively regulate protein kinase signaling cascades, a number of which are implicated in tumorigenesis (6). The lipid phosphatase activity of PTEN dephosphorylates phosphatidylinositol 3,4,5 trisphosphate (PIP3), a potent activator of the protein kinase Akt (7). Loss of PTEN function thus results in the stimulation of cell growth and survival, which are induced by the de-repression of the PI3K/Akt pathway $(8,9)$. However, recent reports suggest that PTEN also has PI3K/Akt-independent activities (10). For example, a phosphatase-inactive PTEN mutant still retains residual tumor suppressive activity, leading to the hypothesis that PTEN has phosphatase-independent tumor suppressor function (11-15).

High-grade malignant gliomas are the most common primary brain tumor in adults. The majority of glioblastomas have histologic characteristics of cells belonging to the astrocytic lineage. Therefore, these tumors are thought to arise from the transformation of astrocytes or their precursors, the neural stem cells (16-19).

The leukemia inhibitory factor receptor $\beta(\operatorname{LIFR} \beta)$ and downstream Stat3 signaling pathway supports the self-renewal proliferation of neural stem cells or stimulates the differentiation of neural stem cells into astrocytes $(20,21)$. The enhanced expression and pro-survival activity of Stat 3 in a subset of glioblastomas supports the concept that the pleiotropic function of Stat 3 signaling contributes to glial cell transformation $(22,23)$. Accordingly, Stat 3 associates with the oncoprotein epidermal growth factor receptor III variant in the nucleus to induce glial transformation, whereas it plays a key role in the PTEN pathway to suppress malignant transformation of astrocytes (24). These observations indicate that Stat 3 plays distinct roles in cell transformation depending on the oncogenic environment.

Recently, there has been a dramatic advance in research on a small subpopulation of cells identified in cancers, which have stem cell properties. The cancer stem cell hypothesis proposes that cancers are derived from a small fraction of cancer stem cells that proliferate through their unique selfrenewal ability (25). Cancer stem cells were first identified in 
leukemia $(26,27)$. Recently, many investigators have identified them in solid tumors, including those of the breast, brain, pancreas, colon and head and neck (28). The cancer stem cell hypothesis established that cancer therapy must be directed against both the resting cancer stem cells and the proliferating cancer cells (29). Therefore, it is essential to understand the major intracellular signaling pathways governing the characteristics of cancer stem cells. It has become clear that PTEN is one of the critical regulators for the development of cancer stem cells and ultimately tumorigenesis.

In this study, we show that ectopic expression of PTEN can disturb Akt and Stat3 phosphorylation, which leads to reduced tumorigenicity both in vitro and in vivo. Furthermore, as demonstrated by the loss of both CD133 expression and neurosphere formation, we found that PTEN can suppress the glioblastoma stem cell (GSC) population. Moreover, PTEN expression also inhibited cell proliferation and induced senescence in glioblastoma. The data suggest that the occurrence of GSCs, and the proliferation and senescence of glioblastoma, which are closely correlated with PTEN expression, are independently regulated by Akt and Stat 3 signaling.

\section{Materials and methods}

Cell culture and genetic modification. Human glioblastomaastrocytoma, epithelial-like cell line U-87MG was purchased from ATCC (www.atcc.org). These cells were maintained in Dulbecco's modified Eagle's medium (DMEM) supplemented with $10 \%$ fetal bovine serum (FBS), $100 \mathrm{U} / \mathrm{ml}$ penicillin, and $100 \mu \mathrm{g} / \mathrm{ml}$ streptomycin (all from Gibco Invitrogen, Carlsbad, CA, www.invitrogen.com). Neurospheres were formed in DMEM containing Nutrient Mixture F-12 (DMEM/F12, Gibco Invitrogen) supplemented with $20 \mathrm{ng} / \mathrm{ml}$ basic fibroblast growth factor (Gibco Invitrogen), $20 \mathrm{ng} / \mathrm{ml}$ recombinant human epidermal growth factor (R\&D Systems, Minneapolis, MN, http://www.rndsystems.com), and $20 \mu \mathrm{l} / \mathrm{ml} \mathrm{N} 2$-supplement (Gibco Invitrogen). A PTEN-expressing plasmid was constructed by cloning PCR-amplified PTEN cDNA into pLPCX (Clontech, Mountain View, CA, http://www.clontech.com). The PTEN-expressing plasmid was transfected into U-87MG cells with Lipofectamine 2000 (Invitrogen) according to the manufacturer's instructions. U-87MG cells that stably express PTEN, by chromosomal integration of the PTEN-expressing plasmid, were selected in DMEM supplemented with $10 \%$ FBS, $100 \mathrm{U} / \mathrm{ml}$ penicillin, $100 \mu \mathrm{g} / \mathrm{ml}$ streptomycin, and $500 \mu \mathrm{g} / \mathrm{ml}$ puromycin (Gibco Invitrogen) for 2 to 3 weeks.

$R N A$ extraction and real-time $R T-P C R$. Total-RNA was extracted using TRIzol (Invitrogen) and 2-5 $\mu \mathrm{g}$ total-RNA was reverse-transcribed using the SuperScriptII ${ }^{\mathrm{TM}}$ First-Strand Synthesis System (Invitrogen) according to the manufacturer's instructions. Real-time RT-PCR was carried out on cDNAs using the QuantiTect SYBR-Green PCR Kit (Qiagen, Valencia, CA). Reactions were conducted in triplicate using the Exicycler $^{\mathrm{TM}} 96$ Real-Time Quantitative Thermal Block (Bioneer, Korea, http:// www.bioneer.co.kr). For quantification, the expression of target genes was normalized against that of the glyceraldehyde 3-phosphate dehydrogenase gene $(G A P D H)$. The PCR primer pairs (sense, antisense) were as follows: $C D 133$ (cag agt aca acg cca aac ca, aaa tca cga tga ggg tca gc) and GAPDH (ggg tgt gaa cca tga gaa, gtc ttc tgg gtg gca gtg at).

Protein extraction and western blot analysis. Cells were washed twice with cold phosphate-buffered saline (PBS), lysed with tissue lysis buffer $(20 \mathrm{mM}$ Tris base $\mathrm{pH} 7.4,137 \mathrm{mM} \mathrm{NaCl}$, 2 mM EDTA, $1 \%$ Triton X-100, 25 mM $\beta$-glycerophosphate, $2 \mathrm{mM}$ sodium pyrophosphate, $10 \%$ glycerol, $1 \mathrm{mM}$ sodium orthovanadate, $1 \mathrm{mM}$ phenylmethylsulfonyl fluoride and $1 \mathrm{mM}$ benzamidine) and clarified by centrifugation at $12,000 \mathrm{x} \mathrm{g}$ for $10 \mathrm{~min}$. Whole-cell extracts were prepared and 20-50 $\mu \mathrm{g}$ protein was resolved by SDS-PAGE and blotted using antibodies against PTEN (sc-6817-R, Santa Cruz Biotechnology), Akt (sc-8312, Santa Cruz Biotechnology), p-Akt (\#4058, Cell Signaling Technology), p-Stat3 (\#9131, Cell Signaling Technology), Stat3 (sc-482, Santa Cruz Biotechnology) and $\beta$-actin (sc-47778, Santa Cruz Biotechnology). Immunoreactivity was detected by enhanced chemiluminescence (Amersham Biosciences).

Immunocytochemical staining. Cells were fixed with $4 \%$ paraformaldehyde in PBS for $20 \mathrm{~min}$ and permeabilized with $0.1 \%$ Triton X-100 in PBS for 15 min. After treatment with $1 \%$ goat serum for $1 \mathrm{~h}$, cells were incubated with primary antibodies against goat anti-human p-Stat3 or CD133 (1:100 dilution) at $4^{\circ} \mathrm{C}$ overnight. Cells were washed with PBS and then incubated with Alexa 488-labeled anti-goat IgG secondary antibody (1:300; Molecular Probes, Eugene, OR) for $2 \mathrm{~h}$.

Senescence analysis. The SA- $\beta$-gal staining method was used to analyze senescence. Cells were washed twice with PBS (pH 7.2), fixed with $2 \%$ formaldehyde and $0.2 \%$ glutaraldehyde in PBS (pH 7.2), and washed in PBS (pH 7.2) supplemented with $1 \mathrm{mM} \mathrm{MgCl}{ }_{2}$. Cells were stained in X-gal solution [1 mg/ml X-gal (Boehringer Ingelheim, Ridgefield, CT, http:/ us.boehringer-ingelheim.com/)], $0.12 \mathrm{mM} \mathrm{K}_{3} \mathrm{Fe}[\mathrm{CN}]_{6}, 0.12 \mathrm{mM}$ $\mathrm{K}_{4} \mathrm{Fe}[\mathrm{CN}]_{6}, 1 \mathrm{mM} \mathrm{MgCl}_{2}$ in PBS at pH 6.0) at $37^{\circ} \mathrm{C}$ overnight and then examined under microscope (CKX41, Olympus, Japan, http://www.olympus-global.com/).

Migration assay. For scratch tests, cells were plated in 6-well plates at 90-100\% confluency. A scratch was made at the center of the well with a 200- $\mu$ l pipette tip. Cell migration was observed under microscope $24 \mathrm{~h}$ after wounding. This experiment was repeated three times.

Rat model of glioblastoma. All of the experimental animals were manipulated in accordance with the guidelines of the CHA University Institutional Animal Care and Use Committee (IACUC090012). Adult male Sprague-Dawley rats $(n=11)$ weighing 260-280 g (Orient, Seoul, Korea) were used in this experiment. One million ordinary U-87 MG cells (called WT thereafter) or U-87 MG cells overexpressing PTEN (called PTEN OE thereafter) in a volume of $2 \mu \mathrm{l}$ were stereotaxically implanted into one hemisphere of the brain $(\mathrm{n}=5 \mathrm{WT}$ and $\mathrm{n}=6$ PTEN OE) using the following coordinates: AP, $+1.0 \mathrm{~mm}$; ML, $-3.0 \mathrm{~mm}$ and DV, $-5.0 \mathrm{~mm}$. Animals were euthanized at 5 weeks following implantation and brains were taken for histological analyses. H\&E staining was carried out on $10 \mu \mathrm{m}$-thick brain sections, whereas immunohistochemical staining was carried out on $40 \mu \mathrm{m}$-thick sections using the following antibodies: 
p-Stat3 (1:100, Cell Signaling), PTEN (1:100, Santa Cruz Biotechnology), human-specific nuclei (hNu) (1:200, Chemicon), goat anti-mouse IgG-conjugated Alexa-555 (1:200, Molecular Probes) and goat anti-rabbit IgG-conjugated Alexa-488 (1:200, Molecular Probes).

Statistical analysis. Graphical data are presented as means \pm SD. Each experiment was performed at least three times and subjected to statistical analysis. Statistically significant differences between two groups were determined using Student's t-test. A p $<0.05$ was considered significant. Statistical analysis was performed using the SAS statistical package vs. 9.13 (SAS Inc., Cary, NC, http://www.sas.com/).

\section{Results and discussion}

PTEN expression results in the depletion of GSCs in glioblastoma. The PTEN pathway is known to control normal stem cell maintenance, self-renewal and migration. PTEN loss confers increased self-renewal capacity to normal stem cells/progenitors, which can cause the development of cancer stem cells and ultimately tumorigenesis. Expression of the GSC tumor marker CD133 was used to examine the effect of PTEN expression on the GSC population. First, the PTEN-deficient U-87MG cells (wild-type, WT) were engineered to stably express PTEN (PTEN-overexpressing, PTEN OE) (Fig. 1A). Fig. 1B shows that the overexpression of PTEN significantly reduced the levels of CD133 RNA and protein, suggesting that PTEN expression resulted in the depletion of GSCs. As confirmation, the loss of further GSC characteristics was analyzed. Cell migration ability is one of the prominent characteristics of glioblastoma cancer stem-like cells (30), and, as expected, PTEN OE cells showed defective migration ability compared to WT (Fig. 1C). In addition, the ability to form neurospheres, another feature of GSCs, was also significantly diminished in PTEN OE cells (Fig. 1D). Taken together, these results suggest that PTEN expression clearly reduced the population of GSCs among U-87MG cells.

PTEN expression induces growth retardation and senescence of glioblastoma cells. Negative regulation by PTEN of PI3K activity and the PI3K/Akt pathway is of critical importance for cell proliferation. To further analyze the tumor suppressive mechanism of PTEN, the effect of PTEN expression on U-87MG cell growth was examined. As expected, cell counting and MTT assay revealed that PTEN expression reduced the proliferation of U-87MG cells (Fig. 2A and B). Cellular senescence, which can be induced by various stimuli, can function as a critical barrier for cancer development and significantly restrict cancer progression. Since proliferation is directly linked to cellular senescence, the effect of PTEN expression on the induction of senescence in U-87MG cells was analyzed. Compared with the WT cells, the number of cells positive for $\beta$-gal staining was significantly increased in PTEN OE at each passage (Fig. 2C). Addition of bpV to the culture medium to inhibit PTEN activity reduced the population of $\beta$-gal-positive cells in PTEN OE, suggesting that cellular senescence is induced by PTEN activity (Fig. 2C). Treatment with bpV consistently rescued the growth retardation of PTEN OE (Fig. 2D). Taken together, these results indicate that the restoration of PTEN activity in glioblastoma cells markedly inhibits cell proliferation and leads to senescence.

PTEN expression in U-87MG cells disrupts Akt and Stat 3 signaling. PTEN inhibits the PI3K/Akt signaling pathway by catalyzing the dephosphorylation of PIP3, while the loss of PTEN induces the activation of the PI3K/Akt cascade, which leads to the stimulation of cell growth and survival $(7,8)$. The LIFR $\beta$-Stat 3 signaling pathway is also regulated by PTEN and functions as a brain tumor suppressor in astrocytes (24). In contrast, Stat 3 activation has been described in a subset of glioblastoma, indicating that Stat 3 plays distinct roles in cell transformation depending on the oncogenic environment $(23,31)$. To examine whether PTEN expression can affect Stat 3 and Akt signaling pathways, the phosphorylation level of Stat 3 and Akt in PTEN OE was compared with that in WT. In response to PTEN expression, the level of phosphoStat3 (p-Stat3) and phospho-Akt (p-Akt) was markedly reduced (Fig. 3A). Dephosphorylation of Stat 3 in PTEN OE was confirmed by immunocytochemical staining (Fig. 3B). Consistent with the result shown in Fig. 1B, the expression level of CD133 was also significantly decreased in PTEN-expressing cells (Fig. 3B). To confirm that the dephosphorylation of Stat3 and Akt was induced by PTEN, the phosphorylation status of STAT3 and AKT was examined in PTEN OE following treatment with bpV for $24 \mathrm{~h}$. As shown in Fig. 3C, chemical inhibition of PTEN activity enhanced Stat3 and Akt phosphorylation, suggesting that Stat 3 and Akt signaling is directly regulated by $P T E N$.

Cell growth, senescence and the population of GSCs are independently regulated by Akt and Stat3 signaling pathways in $U-87 M G$ cells. Since the expression of PTEN in U-87MG cells were shown to modulate Stat 3 and Akt signals, we next examined whether Stat 3 signals are regulated by Akt pathway in U-87MG. To do this, U-87MG cells were treated with PI3K inhibitor LY294002 and, unexpectedly, Stat3 was only transiently dephosphorylated and rapidly restored to its original level within 60 min after LY294002-mediated inhibition of Akt activity (Fig. 4A). This result suggests that Stat3 signal is not a downstream target of Akt pathway in U-87MG. Since PTEN expression resulted in the depletion of the GSC population and caused growth retardation and senescence of U-87MG cells, we speculate that these phenomena might be induced by the disturbance of Akt and Stat3 signaling. To test this hypothesis, Akt activity in U-87MG cells was inhibited by treatment with LY294002 and then the extent of senescence, cell growth and neurosphere formation was examined. As expected, LY294002 treatment specifically induced senescence and severely inhibited cell growth (Fig. 4B and C). In addition, neurosphere formation was completely blocked by the treatment of LY294002 (Fig. 4D). Taken together, these results suggest that the senescence, growth retardation, and depletion of GSCs in U-87MG cells are, at least in part, mediated by the Akt signaling pathway.

Constitutive activation of Stat 3 is frequently detected in clinical samples from a wide range of human carcinomas and the established cancer cell lines, suggesting that Stat 3 activity is crucial to cell survival and growth $(32,33)$. In addition, the JAK/Stat3 signaling pathway is required for the growth of 
A
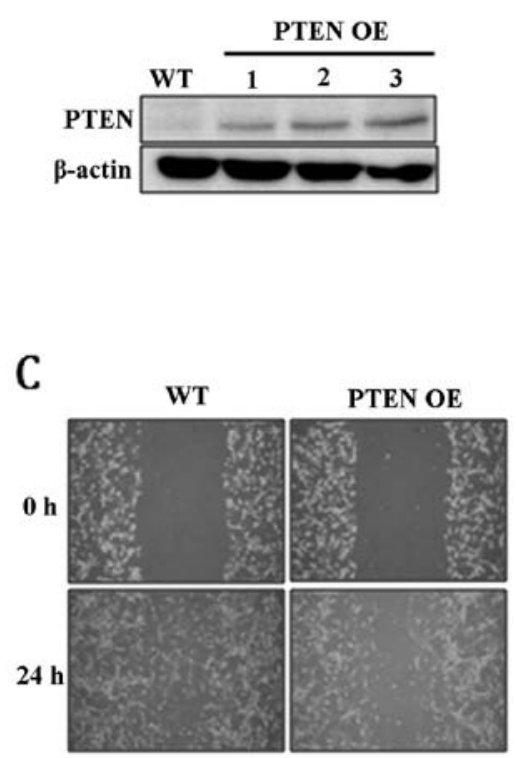

B

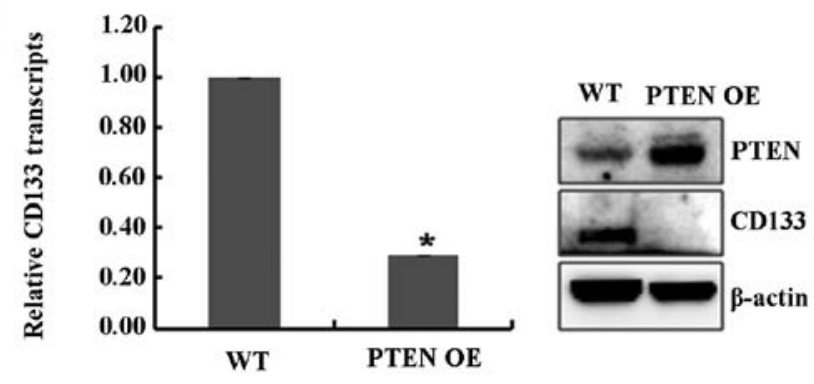

D

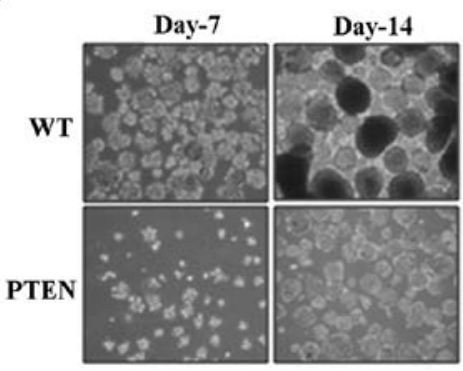

Figure 1. PTEN expression results in depletion of GSCs among glioblastoma cells. (A) PTEN-expressing U-87MG cell lines were constructed by the stable integration of a PTEN-expressing plasmid in U-87MG cells (WT). The expression of PTEN in three stable cell lines (PTEN OE 1,2 and 3) was analyzed by immunoblotting. $\beta$-actin was used as a loading control. (B) CD133 mRNA levels in PTEN OE and WT were compared by real-time RT-PCR and immunoblot analysis. All values are means \pm SD from at least three independent experiments. " $\mathrm{p}<0.05$ based on Student's t-test analysis. (C) Migration of PTEN OE was compared with WT by in vitro scratch wound healing assay. Photographs were taken $24 \mathrm{~h}$ after the wound was made. (D) Representative images demonstrating the reduced neurosphere formation by PTEN OE in comparison to WT. Photographs were taken at 7 and 14 days after the transfer of cells into neurosphere-formation medium.

A

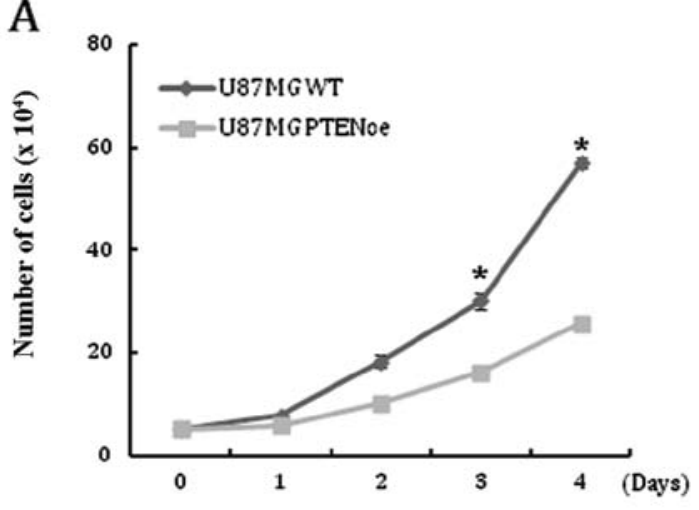

C

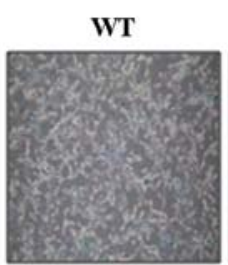

\section{PTEN OE}

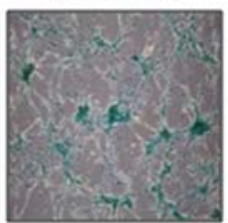

B

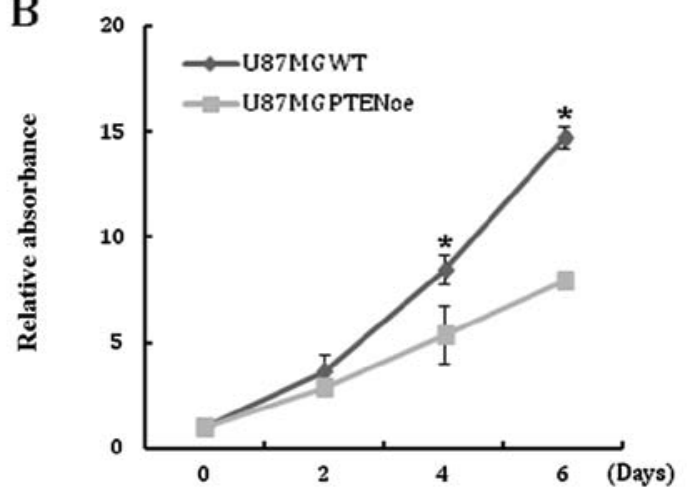

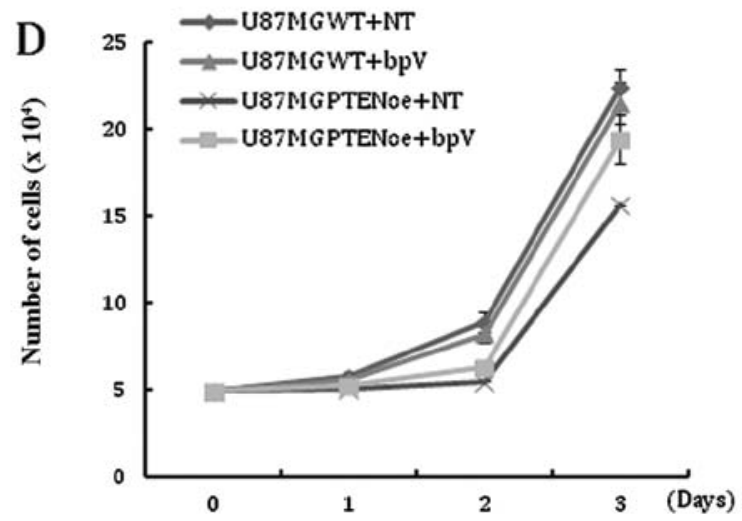

Figure 2. PTEN expression induces growth retardation and senescence of glioblastoma cells. Proliferation of WT and PTEN OE was analyzed by (A) counting cell numbers every $24 \mathrm{~h}$ and by (B) MTT assay every $48 \mathrm{~h}$ under monolayer culture conditions. (C) Staining for senescence-associated acidic $\beta$-galactosidase ( $\beta$-galpositive cells, blue). PTEN OE cells were either untreated (PTEN OE) or pretreated with $2 \mu \mathrm{M} \mathrm{bpV} \mathrm{for} 48 \mathrm{~h}$ before staining (+ bpV). (D) Proliferation of PTEN OE in the presence or absence of $2 \mu \mathrm{M} \mathrm{bpV}$ was examined by counting cell numbers every $48 \mathrm{~h}$. All values are means $\pm \mathrm{SD}$ from at least three independent experiments. $\mathrm{NT}$, not treated; PTEN,PTEN OE. All values are means $\pm \mathrm{SD}$ from at least three experiments. "Indicates significant $(\mathrm{p}<0.05)$ results based on Student's $\mathrm{t}$-test analysis. 

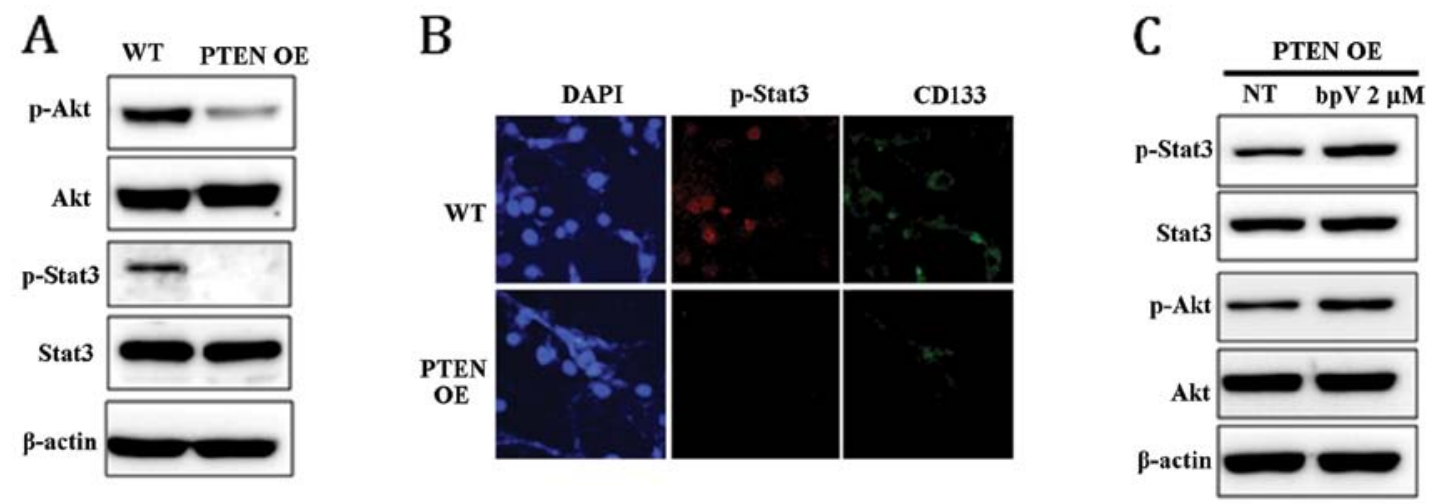

Figure 3. PTEN expression in U-87MG cells disrupts Akt and Stat3 signaling. (A) Levels of p-Stat3 and p-Akt in PTEN OE were compared with WT by immunoblot analysis. $\beta$-actin were used as a loading control. (B) Immunostaining for p-Stat 3 and CD133 expression in WT and PTEN OE. Cells were counter- stained with DAPI. (C) Immunoblot analysis showing p-Stat 3 and p-Akt expression in untreated PTEN OE (NT) or cells cultured in the presence of $2 \mu \mathrm{M}$ bpV for $24 \mathrm{~h}$ $\beta$-actin were used as a loading control.

A
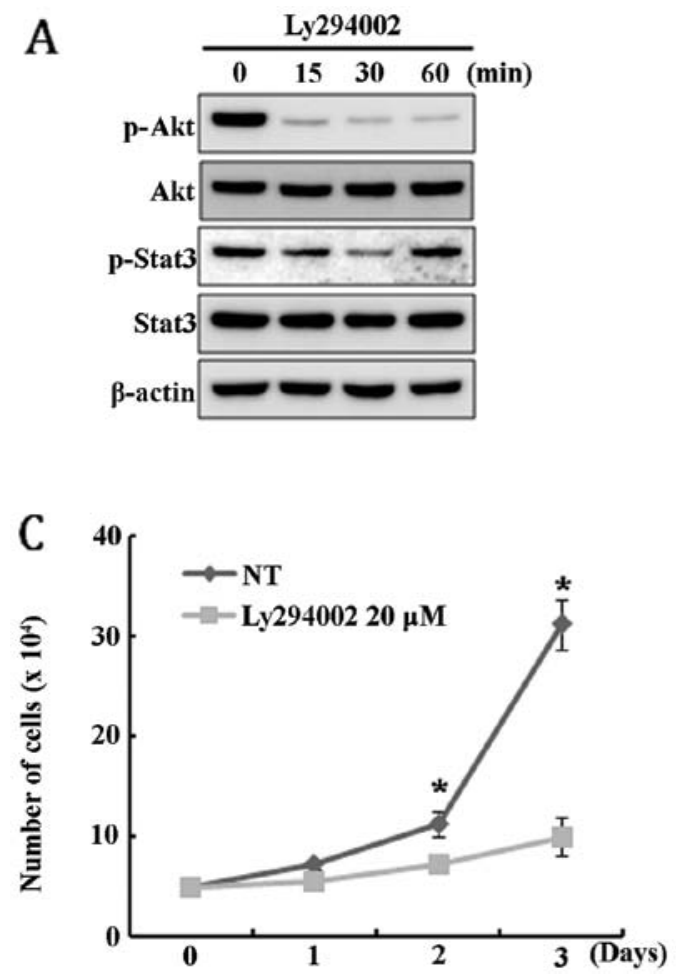

B
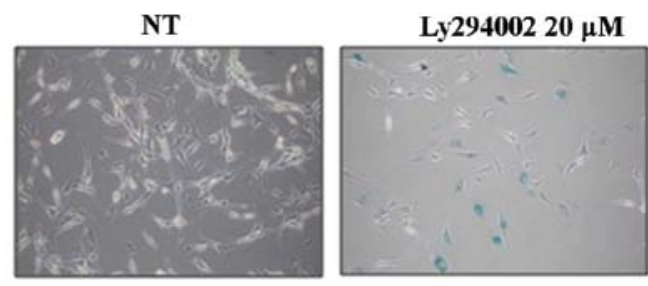

D
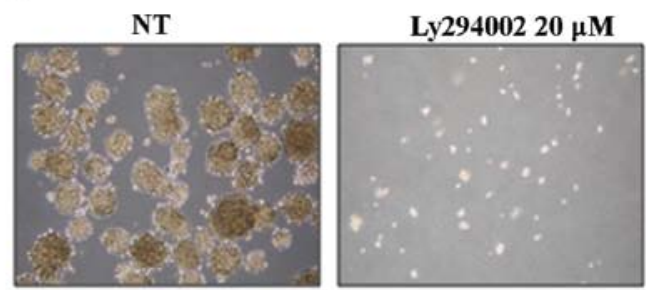

Figure 4. Akt signaling regulates senescence, cell proliferation and neurosphere formation in U-87MG. (A) Immunoblot analysis showing p-Stat3 and p-Akt expression in U-87MG cells harvested at $0,15,30$ and 60 min after treatment with LY294002 (20 $\mu \mathrm{M})$. Akt, Stat3 and $\beta$-actin were used as a loading control. (B) U-87MG cells cultured in the presence or absence of LY294002 $(20 \mu \mathrm{M})$ for 2 days were stained for senescence-associated acidic $\beta$-galactosidase $(\beta$-galpositive cells, blue). (C) Proliferation of U-87MG cells were examined in the presence or absence (NT) of LY294002 (20 $\mu$ M) by counting cell numbers 2 and 3 days after seeding. All values are means \pm SD from at least three experiments. * $p<0.05$ based on Student's t-test analysis. (D) Representative images demonstrating the reduced neurosphere formation of U-87MG cells in the presence of $20 \mu \mathrm{M} \mathrm{LY} 294002$, compared to untreated (NT) group. Photographs were taken at 14 days after the transfer of cells into neurosphere-formation medium.

stem-like cancer cells in various tumors $(34,35)$. For these reasons, we decided to examine the effect of inhibition in Stat 3 signaling on on cell growth, senescence, and neurosphere formation of U-87MG cells. We found that Stat3 was completely dephosphorylated by treatment with $2 \mu \mathrm{M}$ WB1066, while phosphorylation of AKT was not affected (Fig. 5A). Interestingly, we observed that cellular senescence, which can be estimated by $\beta$-gal staining, was not directly induced by inhibiting the Stat 3 signaling pathway, although it caused significant growth retardation (Fig. 5B and C). Treatment with WB1066 completely inhibited the ability of U-87MG to form neurospheres, suggesting that the Stat 3 signaling pathway is required for maintaining the cancer stem-like cell population (Fig. 5D). These results demonstrate that Stat3 signaling regulates proliferation and maintenance of the cancer stem-like cell population but is not involved in the senescence of U-87MG cells. Since PTEN expression in U-87MG disrupts Akt and Stat 3 signals, and that growth retardation, senescence and 
A

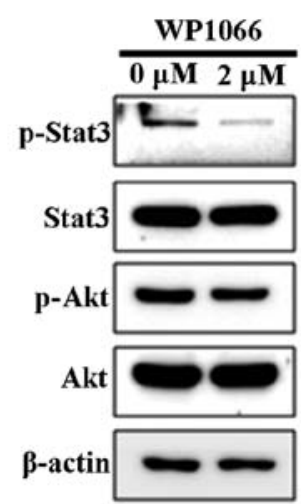

C

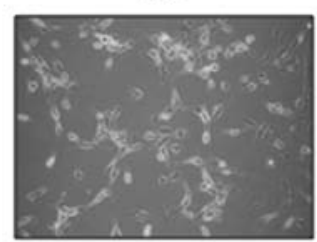

WP1066 $2 \mu \mathrm{M}$

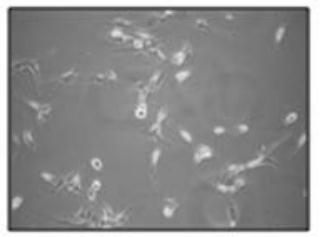

B

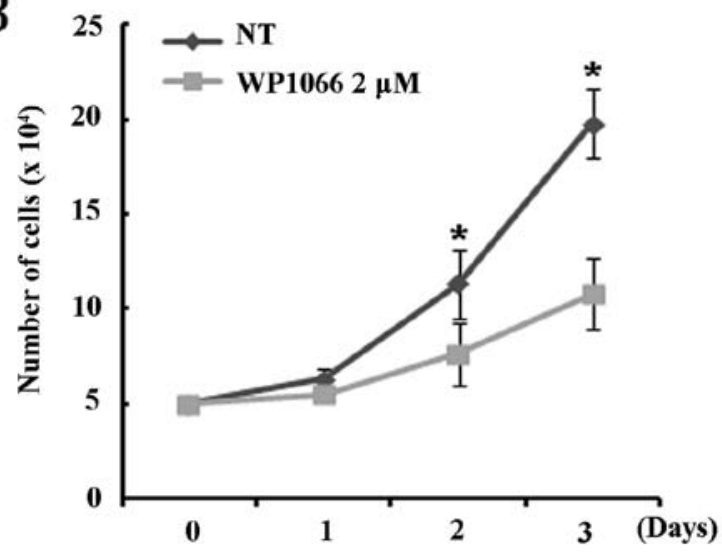

D

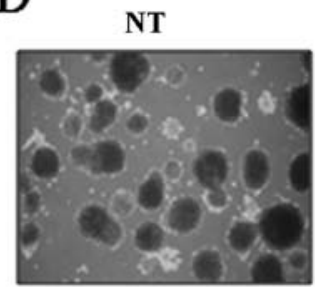

\section{WP1066 $2 \mu \mathrm{M}$}

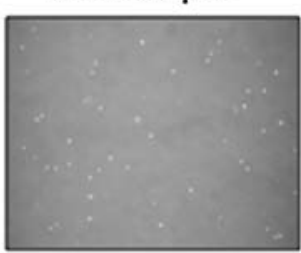

Figure 5. Stat3 signaling regulates cell proliferation and neurosphere formation but not senescence in U-87MG. (A) p-Stat3 and p-Akt expression in U-87MG cells, untreated $(0 \mu \mathrm{M})$ or cultured in the presence of $2 \mu \mathrm{M}$ WP1066 for $48 \mathrm{~h}$, were analyzed by immunoblot. Akt, Stat 3 and $\beta$-actin were used as loading controls. (B) Proliferation of U-87MG cells was examined in the absence (NT) or presence of $2 \mu \mathrm{M}$ WP1066 by counting cell numbers 2 and 3 days after seeding. All values are means \pm SD from at least three experiments. " $p<0.05$ based on Student's t-test analysis. (C) U-87MG cells cultured in the absence (NT) or presence of $2 \mu \mathrm{M}$ WP1066 for 2 days and then stained for senescence-associated acidic $\beta$-galactosidase. (D) Representative images demonstrating the reduced neurosphere formation of U-87MG cells in the presence of $2 \mu \mathrm{M}$ WP1066 compared to untreated (NT) group. Photographs were taken at 14 days after the transfer of cells into neurosphere-formation medium.

A
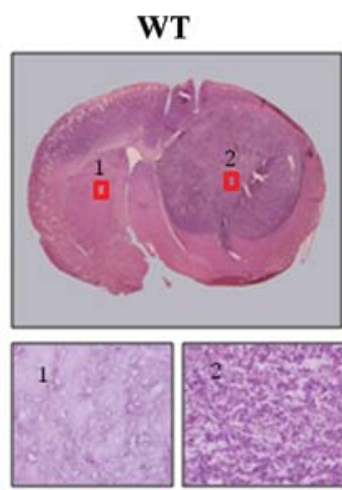

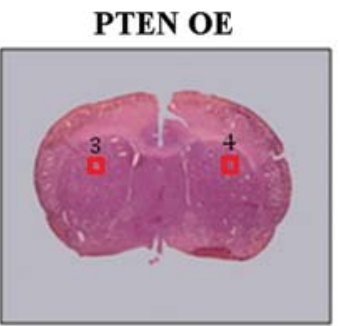

B
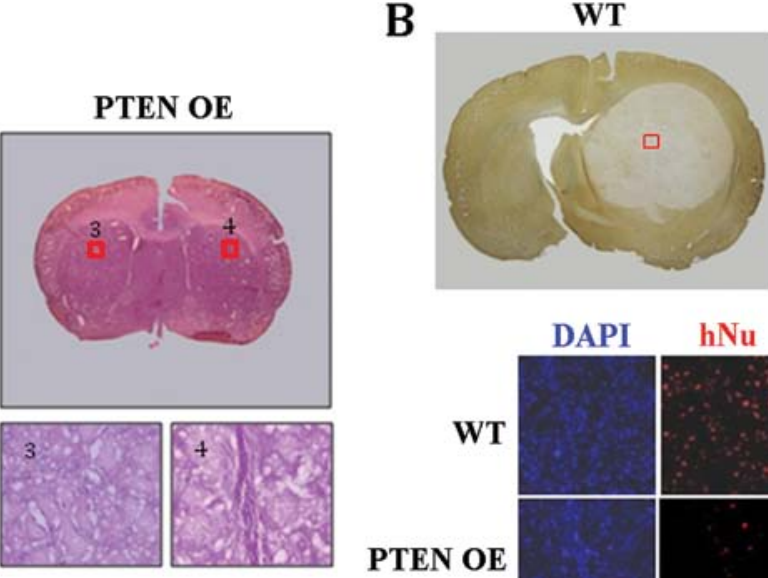
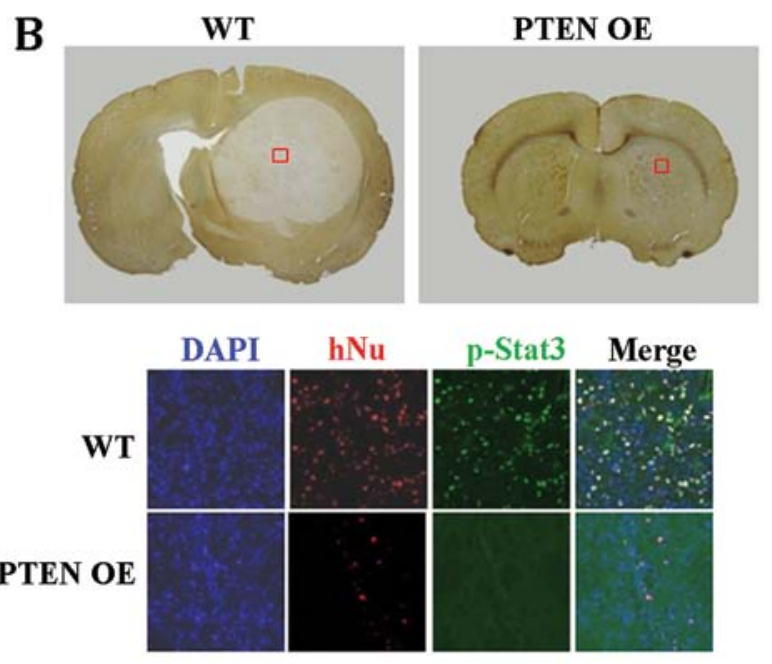

Figure 6. Overexpression of PTEN inhibits tumor formation by U-87MG glioblastoma cells in vivo. (A) H\&E stained sections of brain analyzed at 5 weeks after implantation with WT U-87MG cells or PTEN OE U-87MG cells. Numbers 1-4 correspond to higher magnifications (shown in the panels below) of areas contra-lateral (1 and 3) and ipsi-lateral ( 2 and 4) to the implantation of WT (1 and 2) and PTEN OE cells ( 3 and 4$)$. Note the formation of tumor-like cells in 2, whereas no such cells were formed in 4. (B) Immunohistochemical staining for p-Stat3 at the same stage as in (A). Positive cells were visualized by DAB staining (brown). Note that p-Stat3-positive cells were absent in the area of tumor formation (red box) in WT. Bottom panel shows that hNu- and p-Stat3-positive cells are abundantly detected in WT U87-implanted brain, which are also shown to be co-localized in the Merge image. By contrast, in PTEN OE U-87MG-implanted brain, no p-Stat3-positive cells were found and only a very few hNu-postive cells were detected, indicating that minimal numbers of implanted cells were maintained without forming tumors.

depletion of cancer stem cells are caused by PTEN expression in $\mathrm{U}-87 \mathrm{MG}$, we speculate that PTEN induces growth retardation and cancer stem cell depletion of U-87MG in conjunction with both Stat 3 andAkt signaling pathways, whereas the senescence response of U-87MG cells upon PTEN expression is mainly regulated by the Akt signaling pathway. 
Overexpression of PTEN inhibits tumor formation by $U-87 M G$ glioblastoma cells in vivo. To address whether PTEN is involved in the proliferation of GSCs, PTEN OE or as controls, WT U-87MG cells were implanted into the brain of 8-week-old rats. Strikingly, no tumor-like structures were detected in any of the PTEN OE-implanted group $(\mathrm{n}=6)$, whereas robust tumor-like structures were formed in all of the WT-implanted group $(n=5)$ (Fig. 6A and B). Histological analysis of the WT-implanted brains indicated that the tumor-like structures consisted mainly of undifferentiated glioblastoma cells (Fig. 6A; WT 2). In contrast, such cells were not detected in the PTEN OE-implanted brains (Fig. 6B; PTEN OE 4). Furthermore, immunohistochemical staining revealed that many human-specific nuclear antigen $(\mathrm{hNu})$-positive cells were detected in the WT-implanted brain sections, indicating that U-87MG glioblastoma cells had proliferated extensively after implantation (Fig. 6B; WT). p-Stat3-positive cells were also common in the area of tumor formation (Fig. 6B; WT). In contrast, in the PTEN OE-implanted brains, only a few hNu-positive cells were detected, suggesting that most of the PTEN OE U-87MG glioblastoma cells were not maintained after implantation (Fig. 6B; PTEN OE in the bottom panel). No p-Stat3-positive cells were detected either in the PTEN OE-implanted brains (Fig. 6B; PTEN OE in the bottom panel). Taken together, these results strongly suggest that PTEN negatively regulates the proliferation of GSCs in U-87MG glioblastoma cells, presumably through the inhibition of the Stat 3 pathway.

From our study, we found that analysis of the signaling pathways underlying the tumor suppressor function of PTEN in U-87MG cells can provide experimental evidence how the loss of PTEN promotes pathogenesis of glial tumors. In particular, identification of the Stat 3 and Akt signaling pathways as downstream targets of PTEN provides a mechanism by which PTEN modulates cell proliferation and senescence and maintenance of the GSC population. Accumulating evidence supports the concept that GSCs, which have stem cell-like properties, are the major cause of the occurrence and maintenance of glioblastoma. In this regard, our results will provide important experimental basis for developing therapeutics to treat glioblastoma in the future.

\section{Acknowledgements}

This study was supported by the Korea Science and Engineering Foundation (KOSEF) of the Korean government (MOST) (2010-0003254, 2011-0014084). This study was also supported by Priority Research Centers Program through the National Research Foundation of Korea (NRF) funded by the Ministry of Education, Science and Technology (2009-0093821).

\section{References}

1. Cairns P, Okami K, Halachmi S, Halachmi N, Esteller M, Herman JG, Jen J, Isaacs WB, Bova GS and Sidransky D: Frequent inactivation of PTEN/MMAC1 in primary prostate cancer. Cancer Res 57: 4997-5000, 1997.

2. Feilotter HE, Nagai MA, Boag AH, Eng C and Mulligan LM: Analysis of PTEN and the $10 \mathrm{q} 23$ region in primary prostate carcinomas. Oncogene 16: 1743-1748, 1998.

3. Gray IC, Stewart LM, Phillips SM, Hamilton JA, Gray NE, Watson GJ, Spurr NK and Snary D: Mutation and expression analysis of the putative prostate tumour-suppressor gene PTEN. Br J Cancer 78: 1296-1300, 1998.
4. Li DM and Sun H: TEP1, encoded by a candidate tumor suppressor locus, is a novel protein tyrosine phosphatase regulated by transforming growth factor beta. Cancer Res 57: 2124-2129, 1997

5. Steck PA, Pershouse MA, Jasser SA, Yung WK, Lin H, Ligon AH, Langford LA, Baumgard ML, Hattier T, Davis T, Frye C, Hu R, Swedlund B, Teng DH and Tavtigian SV: Identification of a candidate tumour suppressor gene, MMAC1, at chromosome 10q23.3 that is mutated in multiple advanced cancers. Nat Genet 15: 356-362, 1997.

6. Kolibaba KS and Druker BJ: Protein tyrosine kinases and cancer. Biochim Biophys Acta 1333: F217-F248, 1997.

7. Maehama $T$ and Dixon JE: The tumor suppressor, PTEN/MMAC1, dephosphorylates the lipid second messenger, phosphatidylinositol 3,4,5-trisphosphate. J Biol Chem 273: 13375-13378, 1998.

8. Stambolic V, Suzuki A, de la Pompa JL, Brothers GM, Mirtsos C, Sasaki T, Ruland J, Penninger JM, Siderovski DP and Mak TW: Negative regulation of PKB/Akt-dependent cell survival by the tumor suppressor PTEN. Cell 95: 29-39, 1998.

9. Sun H,Lesche R, Li DM, Liliental J,Zhang H, Gao J, Gavrilova N, Mueller B, Liu X and Wu H: PTEN modulates cell cycle progression and cell survival by regulating phosphatidylinositol 3,4,5,-trisphosphate and Akt/protein kinase B signaling pathway. Proc Natl Acad Sci USA 96: 6199-6204, 1999.

10. Salmena L, Carracedo A and Pandolfi PP: Tenets of PTEN tumor suppression. Cell 133: 403-414, 2008.

11. Blanco-Aparicio C, Renner O, Leal JF and Carnero A: PTEN, more than the AKT pathway. Carcinogenesis 28: 1379-1386, 2007.

12. Georgescu MM, Kirsch KH, Kaloudis P, Yang H, Pavletich NP and Hanafusa $\mathrm{H}$ : Stabilization and productive positioning roles of the C2 domain of PTEN tumor suppressor. Cancer Res 60: 7033-7038, 2000

13. Gildea JJ, Herlevsen M, Harding MA, Gulding KM, Moskaluk CA, Frierson HF and Theodorescu D: PTEN can inhibit in vitro organotypic and in vivo orthotopic invasion of human bladder cancer cells even in the absence of its lipid phosphatase activity. Oncogene 23: 6788-6797, 2004.

14. Koul D, Jasser SA, Lu Y, Davies MA, Shen R, Shi Y, Mills GB and Yung WK: Motif analysis of the tumor suppressor gene MMAC/PTEN identifies tyrosines critical for tumor suppression and lipid phosphatase activity. Oncogene 21: 2357-2364, 2002.

15. Maier D, Jones G, Li X, Schonthal AH, Gratzl O, Van Meir EG and Merlo A: The PTEN lipid phosphatase domain is not required to inhibit invasion of glioma cells. Cancer Res 59: 5479-5482, 1999.

16. Bachoo RM, Maher EA, Ligon KL, Sharpless NE, Chan SS, You MJ, Tang Y, DeFrances J, Stover E, Weissleder R, Rowitch DH, Louis DN and DePinho RA: Epidermal growth factor receptor and Ink4a/Arf: convergent mechanisms governing terminal differentiation and transformation along the neural stem cell to astrocyte axis. Cancer Cell 1: 269-277, 2002.

17. Bajenaru ML, Hernandez MR, Perry A, Zhu Y, Parada LF, Garbow JR and Gutmann DH: Optic nerve glioma in mice requires astrocyte $\mathrm{Nf1}$ gene inactivation and Nf1 brain heterozygosity. Cancer Res 63: 8573-8577, 2003.

18. Holland EC: Gliomagenesis: genetic alterations and mouse models. Nat Rev Genet 2: 120-129, 2001.

19. Uhrbom L, Dai C, Celestino JC, Rosenblum MK, Fuller GN and Holland EC: Ink4a-Arf loss cooperates with KRas activation in astrocytes and neural progenitors to generate glioblastomas of various morphologies depending on activated Akt. Cancer Res 62: 5551-5558, 2002.

20. Bonni A, Sun Y, Nadal-Vicens M, Bhatt A, Frank DA, Rozovsky I, Stahl N, Yancopoulos GD and Greenberg ME: Regulation of gliogenesis in the central nervous system by the JAK-STAT signaling pathway. Science 278: 477-483, 1997.

21. Yoshimatsu T, Kawaguchi D, Oishi K, Takeda K, Akira S, Masuyama N and Gotoh Y: Non-cell-autonomous action of STAT3 in maintenance of neural precursor cells in the mouse neocortex. Development 133: 2553-2563, 2006.

22. Rahaman SO, Harbor PC, Chernova O, Barnett GH, Vogelbaum MA and Haque SJ: Inhibition of constitutively active Stat 3 suppresses proliferation and induces apoptosis in glioblastoma multiforme cells. Oncogene 21: 8404-8413, 2002 . 
23. Weissenberger J, Loeffler S, Kappeler A, Kopf M, Lukes A Afanasieva TA, Aguzzi A and Weis J: IL-6 is required for glioma development in a mouse model. Oncogene 23: 3308-3316, 2004.

24. de la Iglesia N, Konopka G, Puram SV, Chan JA, Bachoo RM You MJ, Levy DE, Depinho RA and Bonni A: Identification of a PTEN-regulated STAT3 brain tumor suppressor pathway. Genes Dev 22: 449-462, 2008.

25. Papagiannakopoulos T and Kosik KS: MicroRNAs: regulators of oncogenesis and stemness. BMC Med 6: 15, 2008.

26. Dick JE: Normal and leukemic human stem cells assayed in SCID mice. Semin Immunol 8: 197-206, 1996.

27. Jin L, Hope KJ, Zhai Q, Smadja-Joffe F and Dick JE: Targeting of CD44 eradicates human acute myeloid leukemic stem cells. Nat Med 12: 1167-1174, 2006.

28. Al-Hajj M and Clarke MF: Self-renewal and solid tumor stem cells. Oncogene 23: 7274-7282, 2004.

29. Rich JN: Cancer stem cells in radiation resistance. Cancer Res 67: 8980-8984, 2007.

30. Inoue A, Takahashi H, Harada H, Kohno S, Ohue S, Kobayashi K, Yano H, Tanaka J and Ohnishi T: Cancer stem-like cells of glioblastoma characteristically express MMP-13 and display highly invasive activity. Int J Oncol 37: 1121-1131, 2010.
31. Wang H, Zhang W, Huang HJ, Liao WS and Fuller GN: Analysis of the activation status of Akt, NFkappaB, and Stat3 in human diffuse gliomas. Lab Invest 84: 941-951, 2004.

32. Buettner R, Mora LB and Jove R: Activated STAT signaling in human tumors provides novel molecular targets for therapeutic intervention. Clin Cancer Res 8: 945-954, 2002.

33. Kusaba T, Nakayama T, Yamazumi K, Yakata Y, Yoshizaki A, Nagayasu T and Sekine I: Expression of p-STAT3 in human colorectal adenocarcinoma and adenoma; correlation with clinicopathological factors. J Clin Pathol 58: 833-838, 2005.

34. Korkaya H, Liu S and Wicha MS: Regulation of cancer stem cells by cytokine networks: attacking cancer's inflammatory roots. Clin Cancer Res 17: 6125-6129, 2011.

35. Marotta LL, Almendro V, Marusyk A, Shipitsin M, Schemme J, Walker SR, Bloushtain-Qimron N, Kim JJ, Choudhury SA, Maruyama R, Wu Z, Gonen M, Mulvey LA, Bessarabova MO, Huh SJ, Silver SJ, Kim SY, Park SY, Lee HE, Anderson KS, Richardson AL, Nikolskaya T, Nikolsky Y, Liu XS, Root DE, Hahn WC, Frank DA and Polyak K: The JAK2/STAT3 signaling pathway is required for growth of CD44CD24 stem cell-like breast cancer cells in human tumors. J Clin Invest 121: 2723-2735, 2011 